\title{
Simulation of the dielectric charging-up effect in a GEM detector
}

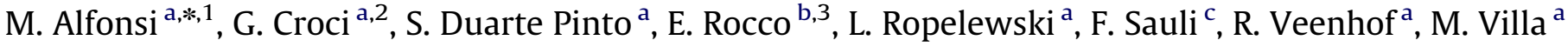 \\ ${ }^{a}$ CERN, Geneva, Switzerland \\ b INFN Torino \& University of Eastern Piedmont \\ c TERA Foundation, Novara, Italy
}

\section{A R T I C L E I N F O}

\section{Article history:}

Received 25 July 2011

Received in revised form

6 December 2011

Accepted 16 December 2011

Available online 24 December 2011

Keywords:

GEM (Gas Electron Multiplier)

Charging up effect

Simulation

Electron transparency

\begin{abstract}
A B S T R A C T
The charging up effect is well-known in detectors containing dielectric materials and it is due to electrons and ions liberated in an avalanche and collected on the dielectric surfaces. In particular in Gas Electron Multiplier (GEM) based detectors, charges can be captured by the Kapton that separates top and bottom electrodes. The collection of a substantial number of charges on the dielectric surfaces induces a modification of the field inside the GEM holes that implies important consequences on some fundamental parameters such as the electron transparency and the effective gain. The correct simulation of this effect opens new ways to the detailed study of the processes that happens in a GEM-based detector and gives the possibility to optimise the GEM geometry in order to avoid it. This paper compares results of the measurements and the simulations, with and without the introduction of the charging-up effect, of the GEM electron transparency in the case of a single GEM detector. The introduction of the charging up effect in the simulation resulted to be crucial in order to get the proper agreement with the measurements. The measurements and simulations of the GEM effective gain will be the subject of a future work.
\end{abstract}

(c) 2011 Elsevier B.V. All rights reserved.

\section{Introduction}

The Gas Electron Multiplier (GEM) [1] charging-up effect consists in a modification of the field configuration due to the accumulation of positive and negative charges, coming either from primary ionisation or liberated in an avalanche process that stick on the insulator surfaces inside the holes. This effect has important consequences on some basic parameters such as the electron transparency and the effective gain, as pointed out in many works (e.g. [2]). On the other hand a detailed description of the process has rarely been taken into account in simulation works found in the literature.

This paper compares results of the measurements and the simulations, with and without the introduction of the charging-up effect, of the electron transparency in case of a single GEM detector. The measurements and simulations of the GEM effective gain will be the subject of a future work. The GEM foils employed in the measurements are the so called "standard GEMs" produced at CERN. ${ }^{4}$ The thickness of the copper clad Kapton polyimide foils is $50 \mu \mathrm{m}$ and the holes, characterised by a bi-conical shape with an internal

\footnotetext{
* Corresponding author.

E-mail address: Matteo.Alfonsi@cern.ch (M. Alfonsi).

${ }^{1}$ Now at NIKHEF, Amsterdam, The Netherlands.

2 Now at CNR-IFP, Milan, Italy.

${ }^{3}$ Now at CERN, Geneva, Switzerland.

${ }^{4}$ Identified in the CERN Materials Store by the codes 08.82.00.100.5 and GEM-100x100-140-70/50-P-U.
}

(external) diameter of $50 \mu \mathrm{m}(70 \mu \mathrm{m})$, are realised over a $140 \mu \mathrm{m}$ pitch hexagonal pattern. All the simulations were performed using the Ansys ${ }^{5}$ software package (electric field map calculation using a Finite Element Method-FEM) and the Garfield [3] program with the recently developed "Microavalanche" algorithm [4]. The gas mixture employed in all measurements and simulations is $\mathrm{Ar} / \mathrm{CO}_{2} 70 \% / 30 \%$.

\section{Measurements of the electron transparency}

In this section the measurements used to validate the simulation results are presented. Fig. 1 shows the sketch of the single GEM detector, provided with a very wide drift gap (13 mm); X-rays enter the detector from the side and convert only in the drift gap creating a primary ionisation current (electrons and ions pairs). Electrons drift towards the GEM holes, where the field is typically high enough to start an avalanche.

In order to study a situation where the GEM foil does not act as a multiplier, a small potential difference was applied on a GEM foil $[5,6]$ (in the case of this study $\Delta V_{G E M}=20 \mathrm{~V}$ ). The measured X-rays interaction rate was around $100 \mathrm{kHz} / \mathrm{mm}^{2}$.

The currents of the four electrodes (Drift, Top GEM, Bottom GEM and Anode) have been measured as a function of the drift field while keeping constant the GEM potential difference and the induction field $\left(\Delta V_{G E M}=20 \mathrm{~V}, E_{\text {Induction }}=3 \mathrm{kV} / \mathrm{cm}\right)$. The behaviour is shown in Fig. 2 .

\footnotetext{
${ }^{5}$ www.ansys.com
} 


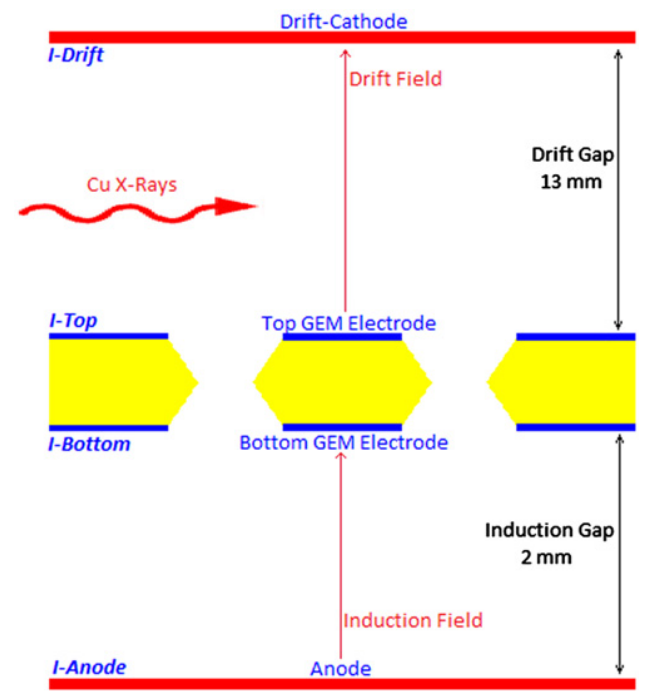

Fig. 1. Experimental setup; X-rays enter from the side and convert only in the wide drift gap.

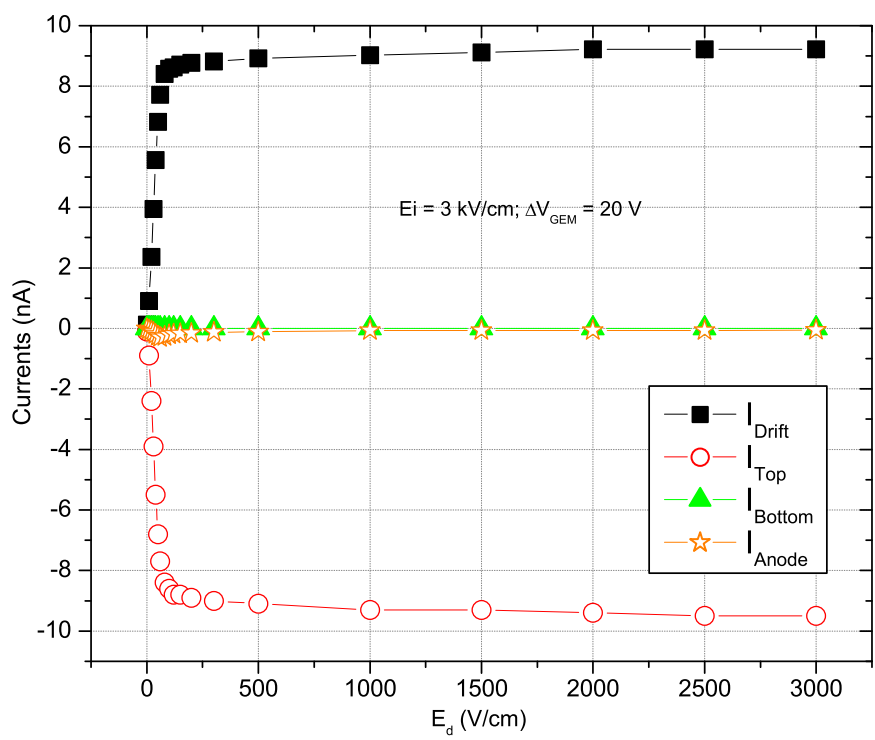

Fig. 2. Drift scan for a small voltage on the GEM.

For drift fields up to $100 \mathrm{~V} / \mathrm{cm}$, recombination between ions and electrons takes place, while, for higher values, the plateau observed in the drift current behaviour proves that all the ionisation current is collected. The measurement shows that, if such a low potential difference is applied, the GEM will be almost opaque for electrons transmission.

The current per hole $\left(I_{\text {hole }}\right)$, a quantity that will be used later in Section 4 to correlate measurements and simulation, can be estimated knowing the irradiated area $A_{\text {Irr }}$ and the value of the ionisation current $\left(I_{i o n}\right)$ as:

$I_{\text {hole }}=\frac{I_{\text {ion }}}{A_{\text {Irr }} \cdot \rho_{\text {Holes }}}$

where $\rho_{\text {holes }}$ is the surface density of holes $\left(N_{\text {holes }} / \mathrm{cm}^{2}\right)$.

\section{Results of the simulation without the charging-up effect}

In the simulation of a single GEM detector, the electron transparency can be evaluated as the ratio between the number
Table 1

Electrons ending place (\%).

\begin{tabular}{lllc}
\hline Top GEM & Kapton & Bottom GEM & Anode \\
\hline $16.6 \%$ & $31.4 \%$ & $0.1 \%$ & $51.9 \%$ \\
\hline
\end{tabular}

of electrons that are able to reach the anode and the number of electrons generated in the drift gap. The simulation of a GEM foil in a configuration with $\Delta V_{G E M}=20 \mathrm{~V}, E_{\text {Drift }}=100 \mathrm{~V} / \mathrm{cm}$ and $E_{\text {Ind }}=3 \mathrm{kV} / \mathrm{cm}$ has been performed generating 2000 electrons in the drift gap, $300 \mu \mathrm{m}$ above the top GEM electrode, and recording the position of each electron at the end of the drift path: the final position allows to infer if the electron has been collected by the Top or Bottom GEM electrode, the Kapton surface inside the hole, or it has reached the anode. Table 1 summarises the result for this electrostatic configuration.

Around $50 \%$ of the electrons can pass through the hole and is collected on the anode electrode. In this simulation, the GEM is not opaque for electrons transmission: the discrepancy between measurements and simulations is apparent.

\section{Simulation of the GEM charging-up}

In order to include the charging-up effect in a GEM simulation, the procedure should evaluate the charges accumulated in the dielectric surfaces and accordingly correct the electric field in the gas volume. So the evolution of the accumulated charge has been divided into discrete steps: after each step, the electric field map has been again calculated taking into account the new values of the surface charge. An equilibrium configuration is expected when no further electrons are impinging on the dielectric surface: even if in a real situation the surface charge slowly move towards the electrodes, the Kapton resistivity is extremely high and this effect can be neglected in the simulation.

In this work the distribution of the charges on the Kapton surface inside the holes has been approximated. The axial symmetry and the bi-conical shape of the holes suggest the partition of the surface into a top Kapton half $\left(k_{t}\right)$ and a bottom Kapton half $\left(k_{t}\right)$, closer to the Top and the Bottom GEM electrode respectively; inside each half, the charge distribution is considered as uniform and the electrons impinging on that contribute to the whole charge independently from the precise impact spot. Finer partition and the impact on the results can be the subject of a future work.

A schematic description of such an iterative procedure is the following:

1. A simulation of 2000 electrons is started in an electrostatic configuration without any charge on the Kapton surfaces.

2. The fraction of charges collected on Anode, bottom GEM electrode, bottom Kapton half, top Kapton half and top GEM electrode $\left(N \%_{\text {end-layer }}\right)$ is evaluated.

3. A charge deposited onto the Kapton surfaces $\left(q_{a d d-t K}, q_{a d d-b K}\right)$ is computed from the fractions using the normalisation factor $I_{\text {hole }}$ as follows:

$q_{\text {add-t(b)K}}[C]=N \%_{t(b) K}[\#] \cdot I_{\text {hole }}[A] \cdot t_{\text {step }}[s]$

4. This charge is added on top (bottom) Kapton and a new electric field map is calculated.

5. Another simulation of 2000 electrons using the new electric field map is started. 


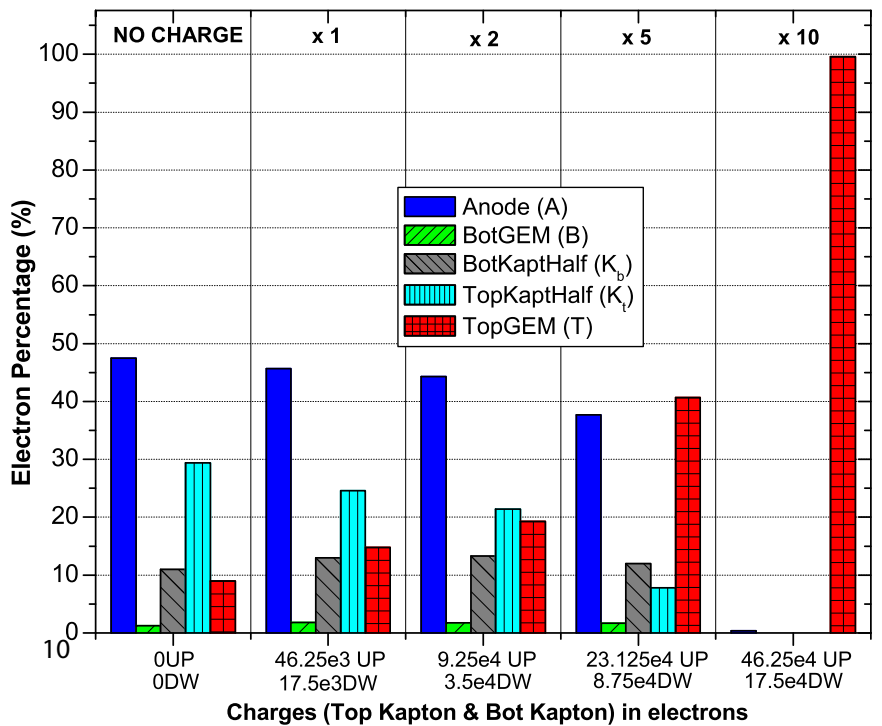

Fig. 3. Optimisation of time iteration step. More details in the text.

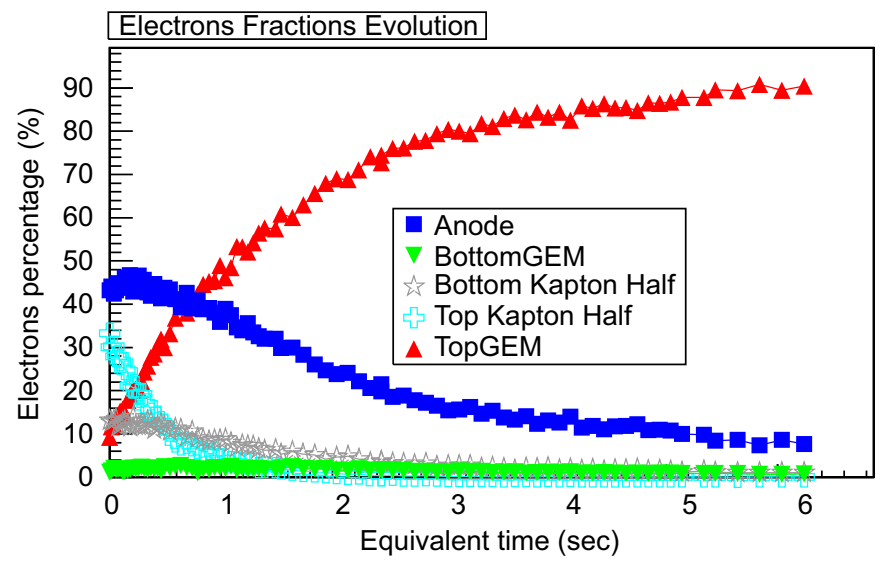

Fig. 4. Electron fractions collection on different layers as a function of elapsed time; $\Delta V_{\text {GEM }}=20 \mathrm{~V}, E_{\text {Drift }}=100 \mathrm{~V} / \mathrm{cm}$ and $E_{\text {Ind }}=3 \mathrm{kV} / \mathrm{cm}$.

An arbitrary factor $t_{\text {step }}$ measured in seconds is present in Eq. (2) and it is used to correlate the simulation with the measurement. The effects of its choice and how it has been chosen are described in the following paragraphs.

\subsection{The effect of the $t_{\text {step }}$ parameter}

The charge to be added in each step linearly depends on the $t_{\text {step }}$ parameter (see Eq. (2)), which can be arbitrarily chosen. In order to evaluate the effect of this parameter, a preliminary check in the first steps of the simulation has been performed. In Fig. 3, the fractions of electrons that were collected, after the initial step, in the different detector layers are shown in the leftmost column. This distribution has been employed in different simulations of the second step using four different values of $t_{\text {step }}$. The variation of the fractions after the second step is shown in the other columns of the figure. The value of $t_{\text {step }}$ corresponding to the second column has been arbitrarily defined as $0.1 \mathrm{~s}$ as a reference. If $t_{\text {step }}$ has a too large value (as in the case of the last column), a very high amount of charge will be added in one step and the discretisation of the charging-up process, which is by nature continuous, fails. Therefore, $t_{\text {step }}$ should be chosen in such a way that a small amount of charge is added in each iteration and that the electron distribution does not sharply change.
Table 2

Charge corresponding to different values of equivalent time.

\begin{tabular}{lll}
\hline Equivalent time $(\mathbf{s})$ & $K_{t}$ Charge $\left(\mathrm{e}^{-}\right)$ & $K_{b}$ Charge $\left(\mathrm{e}^{-}\right)$ \\
\hline $\mathbf{0 . 1}$ & $4.625 \times 10^{4}$ & $1.75 \times 10^{4}$ \\
$\mathbf{0 . 5}$ & $18.39 \times 10^{4}$ & $11.8 \times 10^{4}$ \\
$\mathbf{1}$ & $23.2 \times 10^{4}$ & $19.32 \times 10^{4}$ \\
$\mathbf{2}$ & $26.26 \times 10^{4}$ & $29.1 \times 10^{4}$ \\
$\mathbf{3}$ & $23.85 \times 10^{4}$ & $35.14 \times 10^{4}$ \\
$\mathbf{4}$ & $27.04 \times 10^{4}$ & $38.63 \times 10^{4}$ \\
\hline
\end{tabular}

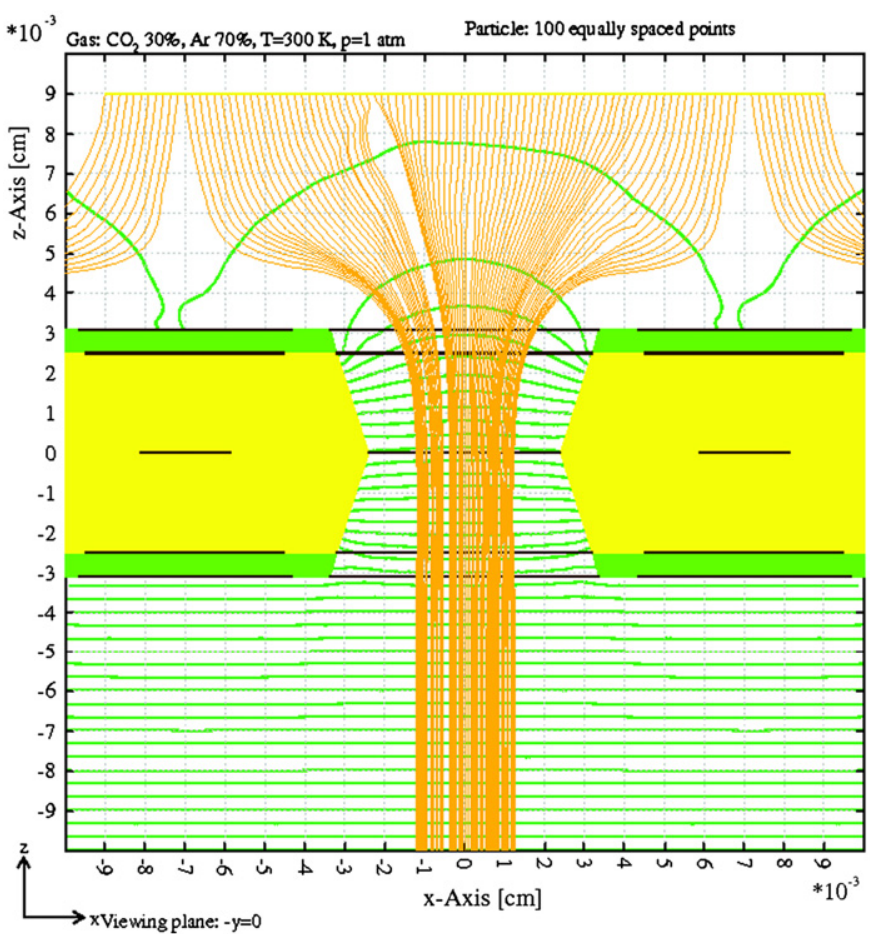

Fig. 5. Drift path (without diffusion) of electrons starting $90 \mu \mathrm{m}$ above the GEM in an electrostatic configuration without charges on Kapton surface (equivalent time $=0 \mathrm{~s}$ ).

\subsection{Results of the simulation including the charging-up effect}

The described procedure has been applied to the simulation of the electrostatic configuration with $\Delta V_{G E M}=20 \mathrm{~V}, E_{\text {Drift }}=100 \mathrm{~V} / \mathrm{cm}$ and $E_{\text {Ind }}=3 \mathrm{kV} / \mathrm{cm}$, that is characterised by a small measured electron transparency (see Fig. 2).

Besides, the algorithm follows other two prescriptions:

1. If the relative statistical error (error/value) of the electron distribution on top (bottom) Kapton is higher than a userdefined threshold ( $2 \%$ in the case of this study), other 2000 primary electrons are generated using the same electrostatic configuration, in order to increase statistics. The error is calculated according to the binomial statistics.

2. A minimum and a maximum threshold have been established for the charge that has to be added in each iteration: if the calculated charge is above or below these thresholds, the charge is scaled by increasing or reducing the time step.

Fig. 4 shows the evolution of the electron fractions collected in the different layers as a function of elapsed time. The $x$-axis coordinate is referred as equivalent time because every elapsed time value corresponds to a specific equivalent amount of charge 


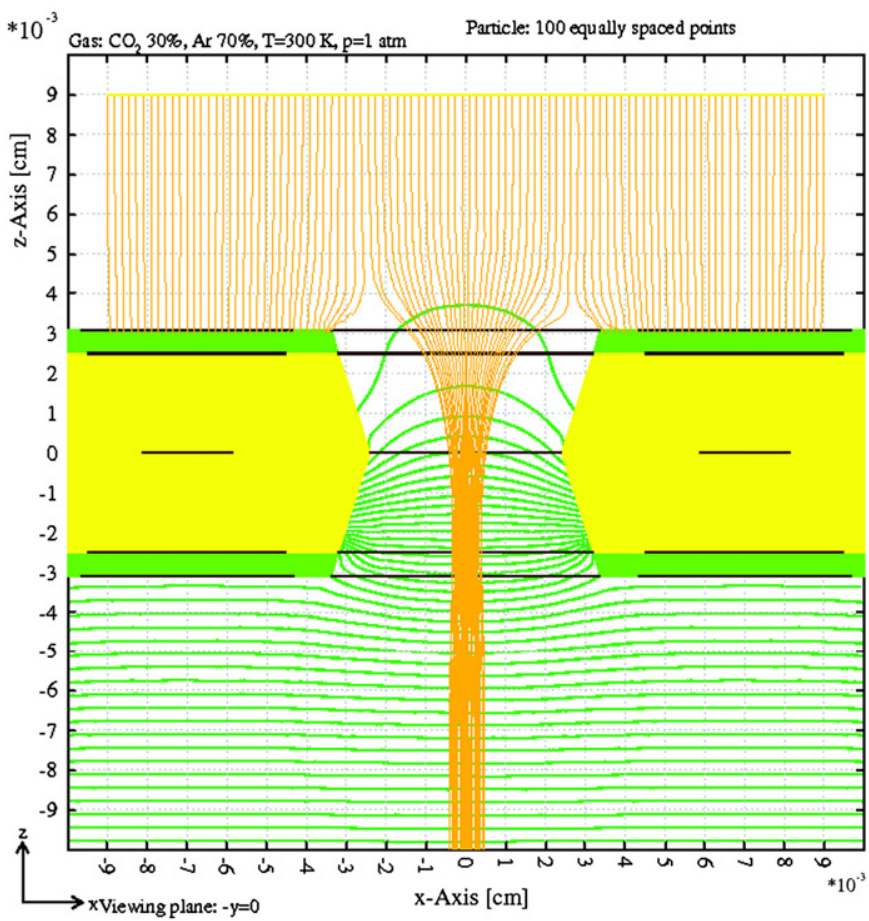

Fig. 6. Drift path (without diffusion) of electrons starting $90 \mu \mathrm{m}$ above the GEM, in an electrostatic configuration with accumulated charges on Kapton surface (equivalent time $=4 \mathrm{~s}$ ).

collected on the Kapton; the values of the collected charge for different equivalent times is reported in Table 2.

At the beginning of the process, half of the generated electrons reaches the anode and only $10 \%$ ends up on the top GEM electrode.

The charging-up cannot be neglected because $30 \%$ of the charge is captured by the top Kapton half and around 10\% is collected by the bottom Kapton half. When a large amount of charge is accumulated, the GEM becomes more and more opaque: at the end of the process the top GEM electron fraction reaches a value around $85 \%$, while the anode electrons fraction reduces to $10 \%$. In addition, as the Kapton charges-up, a lower number of electrons is able to end up on dielectric surfaces; at the end of the process, the fraction of charges collected by the insulator is almost zero.

Figs. 5 and 6 show the variation of the equipotential lines and of the electron drift lines respectively at the beginning (equivalent time $=0 \mathrm{~s}$ ) and when the process is well advanced (equivalent time $=4 \mathrm{~s}$ ): a higher number of electrons is able to enter the hole when Kapton surfaces are not charged.

The results of the simulations are able to reproduce the measurements (see Fig. 2), demonstrating that the introduction of the charging-up effect is crucial in order to correctly simulate the GEM electron transparency.

\section{Conclusions and future plans}

The correct simulation of the charging-up effect opens new ways to the detailed study of the processes that happens in a GEM-based detector and gives the possibility to optimise the GEM geometry in order to avoid it. The described procedure introduces the charging-up effect in the simulation of the electron transparency of a single GEM detector, demonstrating that a proper agreement between measurements and simulations can not be achieved if this effect is neglected.

A future work will also study the impact of the charging up effect in the simulation of the GEM effective gain. Moreover, a new field solver called NeBEM [7] has recently been developed: it does not use FEMbut exploits the so called "Boundary Element Method" (BEM), resulting more precise in the calculation of the field near the edges compared to a standard FEM method. All the described simulations will be repeated exploiting this new tool.

\section{References}

[1] F. Sauli, Nuclear Instrumentation and Methods in Physics Research Section A 386 (1997) 531.

[2] W. Anderson, et al., in: IEEE Nuclear Science Symposium 2006 Conference Records, 2007, pp. 3847-3851.

[3] R. Veenhof, Nuclear Instrumentation and Methods in Physics Research Section A 419 (1998) 726.

[4] 〈http://garfield.web.cern.ch/garfield/〉.

[5] F. Sauli, L. Ropelewski, P. Everaerts, Nuclear Instrumentation and Methods in Physics Research Section A 560 (2006) 269.

[6] G. Croci, M. Alfonsi, S. Duarte Pinto, L. Ropelewski, F. Sauli, M. Villa, E. Rocco, Journal of Instrumentation 5 (2010) P03001.

[7] N. Majumdar, S. Mukhopadhyay, Journal of Instrumentation 9 (2007) P09006. 